\title{
Arbitrage and viability in securities markets with fixed trading costs
}

\author{
Elyès Jouini* \\ Clotilde Napp
}

\author{
July, 1999
}

\begin{abstract}
This paper studies foundational issues in securities markets models with fixed costs of trading, i.e. transactions costs that are bounded regardless of the transaction size, such as: fixed brokerage fees, investment taxes, operational and processing costs, or opportunity costs. We show that the absence of free lunches in such models is equivalent to the existence of a family of absolutely continuous probability measures for which the normalized securities price processes are martingales, conditional on any possible future event. This is a weaker condition than the absence of free lunches in frictionless models, which is equivalent to the existence of an equivalent martingale measure. We also show that the only arbitrage free pricing rules on the set of attainable contingent claims are those that are equal to the sum of an expected value with respect to any absolutely continuous martingale measure and of a bounded fixed cost functional. Moreover, these pricing rules are the only ones to be viable as models of economic equilibrium.

Keywords :arbitrage - fixed costs - absolutely continuous martingale measure - contingent claims pricing - viability

${ }^{*}$ CREST-ENSAE, 15, Bd Gabriel Péri, 92241 Malakoff Cedex, France and CERMSEMUniversité de Paris I. Jouini is currently visiting the Stern School of Business at NYU, 44

${ }^{\dagger}$ Salomon Brothers, 111 Buckingham Palace Road, London, SW1W OSB, U.K. Salomon is not responsible for any statement or conclusions herein, and no opinions, theories, or techniques presented herein in any way represent the position of Salomon Brothers Inc.

${ }^{\ddagger}$ CREST, 15, Bd Gabriel Péri, 92241 Malakoff Cedex, France.
\end{abstract} W 4th St, NY, 


\section{Introduction}

The Fundamental Theorem of Asset Pricing, which originates in the ArrowDebreu model (Debreu [1959]) and is further formalized in (among others) Cox and Ross (1976), Harrison and Kreps (1979), Harrison and Pliska (1981), Duffie and Huang (1986), Dybvig and Ross (1987), Dalang, Morton and Willinger (1989), Back and Pliska (1990), and Delbaen and Schachermayer (1994), asserts that the absence of free lunch in a frictionless securities market model is equivalent to the existence of an equivalent martingale measure for the normalized securities price processes. The only arbitrage free and viable pricing rule on the set of attainable contingent claims, which is a linear space, is then equal to the expected value with respect to any equivalent martingale measure.

In this paper, we study some foundational issues in the theory of asset pricing in securities markets models with fixed trading costs. Transaction costs are said to be fixed in the sense that they are bounded regardless of the transaction size. Such fixed costs include for example fixed brokerage fees, brokerage arrangements where marginal fees go to zero beyond a given volume that is reset periodically (such arrangements are common in the industry), fixed investment taxes to gain access to a market (such as a foreign market), operational and processing costs that typically exhibit strong economies of scale (e.g. through automation), fixed costs involved in setting up an office and obtaining access to information, and the opportunity cost of looking at a market or of doing a specific trade. We find that the absence of free lunches in models with fixed trading costs is equivalent to the existence of a family of "absolutely continuous" probability measures ${ }^{1}$ for which the normalized (by a numeraire) securities price processes are martingales, conditional on any possible future event. Note that this is a weaker condition than the existence of an equivalent martingale measure (as in frictionless markets) because in this case the martingale measures are only required to be absolutely continuous. As in the Fundamental Theorem of Asset Pricing, we find that the absence of free lunches is also equivalent to the existence of a family of nonnegative state price densities and to the existence of a family

\footnotetext{
${ }^{1}$ Let $(\Omega, F, P)$ be a given probability space. We say that another probability measure $Q$ defined on the same probability space $\hat{e}(\Omega, F, P)$ is absolutely continuous with respect to $P$, and we shall write $Q<<P$, if for all event $A$ in $F$ satisfying $P(A)=0$ we have $Q(A)=0$.
} 
of continuous weakly positive linear operators. We define admissible pricing rules on the set of attainable contingent claims as the price functionals that are arbitrage free and are lower than or equal to the surreplication cost (i.e. the lowest cost of dominating a given payoff). Indeed, no rational agent would pay more than its surreplication cost for a contingent claim since there is a cheaper way to achieve at least the same payoff using a trading strategy. We then show that the only admissible pricing rules on the set of attainable contingent claims are those that are equal to the sum of an expected value with respect to any absolutely continuous martingale measure and of a bounded fixed cost functional. Moreover, these pricing rules are the only ones to be viable as models of economic equilibrium, i.e. such that there exist some price-taking maximizing agents who are happy with their initial endowment, and hence for whom supply is equal to demand.

A simple example can illustrate our main result. Consider a model where two securities, denoted by $A$ and $B$, can be traded at two dates 0 and 1 and in two possible states of the world $s_{1}$ and $s_{2}$ at date 1 . Security $A$, the numeraire, is normalized to be always worth one unit of account and security $B$ has a value of 1 at date 0 and a value of 1 or 2 at date 1 in state $s_{1}$ or $s_{2}$ respectively (all in numeraire units). In the perfect market case, this model yields an arbitrage opportunity which consists in buying one unit of $B$ and selling one unit of $A$ at date 0 at a zero investment cost, and closing the position at date 1 at a profit in state $s_{1}$ and at no loss in state $s_{2}$. If we introduce fixed trading costs, this arbitrage opportunity disappears since the investment required at date 0 by the strategy is not zero anymore but is equal to the fixed cost. According to the Fundamental Theorem of Asset Pricing, there cannot exist an equivalent martingale measure. Nevertheless, the probability $Q$ defined on the set $S=\left\{s_{1}, s_{2}\right\}$ of the possible states of the world at date 1 by $Q\left(s_{1}\right)=1$ and $Q\left(s_{2}\right)=0$ is an absolutely continuous martingale measure for securities $A$ and $B$.

There is an existing body of literature that studies transaction costs and other market frictions. For instance, Jouini and Kallal (1995a) studies proportional transaction costs and finds that a bid-ask price process is arbitrage free if and only if there exists an equivalent probability measure that transforms some process between the normalized bid and ask price processes into a martingale. Jouini and Kallal (1995b) studies the case of short sales constraints and shortselling costs (as well as different borrowing and lending rates) and finds that the absence of arbitrage is equivalent to the existence 
of an equivalent supermartingale measure. The set of expected values of the payoff of a contingent claim with respect to all the martingale (resp. supermartingale) measures is an interval and coincides with the set of its possible prices compatible with arbitrage and economic equilibrium. The characteristic of this class of frictions is that they lead to a pricing rule that is sublinear, i.e. positively homogeneous and subadditive, and since this is not the case for fixed transaction costs they require a specific analysis. Also, Cvitanic and Karatzas (1993 and 1996) study the optimal hedging problem in a diffusion model with portfolios constrained to belong to a given convex set and proportional transaction costs respectively. Pham and Touzi (1996) studies the case of constraints that take the form of closed convex cones in finite discrete time. ${ }^{2}$ As far as fixed transaction costs are concerned Duffie and Sun (1990), Grossman and Laroque (1990) and Morton and Pliska (1995), among others, have studied the optimal portfolio problem with transaction fees that are proportional to the size of the overall portfolio (as opposed to the size of the specific transaction).

The remainder of the paper is organized as follows. Section 2 describes our securities markets model with fixed trading costs. Section 3 characterizes the absence of free lunches in such a model. Section 4 characterizes the arbitrage free and viable pricing rules. Section 5 concludes.

\section{The model with fixed costs}

The securities market model consists of a set $F=[0, T]$ of trading dates, where $T$ denotes the terminal date for all economic activity; a complete probability space $(\Omega, F, P)$, where the set $\Omega$ represents all possible states of the world; an information structure which describes how information is revealed to agents, given by a filtration $F=\left\{F_{t}\right\}_{t \in T}$ with $F_{0}=\{\emptyset, \Omega\}$ and $F_{T}=F ; n+1$ traded securities $0, \ldots, n$ and a $(n+1)$-dimensional, $F$ adapted process $Z=\left\{Z_{t} ; t \in \mp\right\}$ with component processes $Z^{0}, \ldots, Z^{n}$ where $Z_{t}^{k}$ represents the price of security $k$ at time $t$. We assume that for all $t$, $Z_{t}^{0}=1$, which means that the riskless rate is equal to zero. Note that this assumption amounts to a normalization of all securities prices by a numeraire,

\footnotetext{
${ }^{2}$ Other papers on market frictions include Magill and Constantinides (1976), Constantinides (1986), Dybvig and Ross (1986), Prisman (1986), Ross (1987), Taksar et al. (1988), He and Pearson (1991), Bensaid et al. (1992), Hindy (1995) and Jouini and Kallal (1999).
} 
and can be made without any loss of generality as long as at least one of the securities has a positive price at any time. In the remainder of the paper we shall refer to the 0th security as the riskless asset. We also make the technical assumption ${ }^{3}$ that for any trading date $t$ in $F, Z_{t}$ is in $L^{1}\left(\Omega, F_{t}, P\right)$.

A trading strategy is a $(n+1)$-dimensional $F$-adapted process $\theta=$ $\left\{\theta_{t} ; t \in \mp\right\}$ with component processes $\theta_{t}^{0}, \ldots, \theta_{t}^{n}$ where $\theta_{t}^{k}$ represents the quantity of security $k$ held at time $t$. The vector $\theta_{t}$ represents the agent's portfolio at time $t$ and its components may take negative as well as positive values. Hence, ${ }^{4} V_{t}^{\theta}=\theta_{t} \cdot Z_{t}$ is the market value of the portfolio $\theta_{t}$ at date $t$ and we call the process $V^{\theta}=\left\{V_{t}^{\theta} ; t \in \mp\right\}$ the value process for the strategy $\theta$. Let $\bar{\theta}_{t}$ denote for each date $t$ the vector $\left(\theta_{t}^{1}, \ldots, \theta_{t}^{n}\right)$ of quantities of risky securities held at time $t$. As in Harrison and Kreps (1979), we only consider simple strategies, i.e., strategies such that: for all $t, \theta_{t} \cdot Z_{t}$ is in $L^{1}\left(\Omega, F_{t}, P\right)$; agents may trade only at a finite number of dates (although that number can be arbitrarily large) that must be specified in advance. ${ }^{5}$ Note that simple strategies are natural in our context because we shall assume that agents incur a fixed transaction cost each time they trade.

We denote by $c_{t}$ the positive fixed transaction cost paid at date $t$ if trading has occurred in any of the risky securities and $c=\left\{c_{t} ; t \in \mp\right\}$. If agents do not trade in any of the risky securities at time $t$, then we assume that they do not incur any transaction cost. The transaction cost is fixed in the sense that it is bounded regardless of the amount of securities traded, i.e. for all $t$ there exists some real number $C_{t}$ such that $0<c_{t}<C_{t} P$ a.s.. We assume that the process $c$ is $F$-adapted, which means that agents only know at time $t$ the past and current values of the fixed trading cost but nothing more. We also allow the fixed transaction costs to depend upon the trading strategy (and not to be necessarily strictly positive at each trading date), i.e., to each simple strategy $\theta$ with trading dates $t_{0}, \ldots, t_{N}=T$ is associated a nonnegative transaction cost process $c^{\theta}=\left(c_{t}^{\theta}\right)_{t \in\left\{t_{0}, \ldots, t_{N}\right\}}$ with $c_{t}^{\theta}=C\left(t,\left(\theta_{t^{\prime}}\right)_{t^{\prime} \leq t}\right)$ such that:

- for any simple trading strategies $\theta$ and $\theta^{\prime}$, such that $\bar{\theta}=\overline{\theta^{\prime}}$, we have $c^{\theta}=c^{\theta^{\prime}}$ and agents do not pay any fixed transaction cost if they do not

\footnotetext{
${ }^{3}$ We recall that $L^{1}(\Omega, F, P)$ denotes the set of measurable random variables with finite expected value with respect to $P$.

${ }^{4}$ For all $(x, y)$ in $R^{d} \times R^{d}$ for some positive real number $d$, we let $x \cdot y=\sum_{i=1}^{d} x_{i} y_{i}$.

${ }^{5}$ The extension to trading dates that are stopping times (instead of being specified in advance) is straightforward.
} 
trade the risky securities, i.e. for any simple strategy $\theta$ with trading dates $t_{0}, \ldots t_{N}$,

$$
\begin{aligned}
c_{t_{i}}^{\theta} 1_{\left(\bar{\theta}_{t_{i}}=\bar{\theta}_{t_{i-1}}\right)} & =0 \text { for all } i \text { with } 1 \leq i \leq N \\
c_{t_{0}}^{\theta} 1_{\left(\bar{\theta}_{t_{0}}=0\right)} & =0, c_{T}^{\theta} 1_{\left(\bar{\theta}_{T}=0\right)}=0 \\
c_{t}^{\theta} & =0 \text { for all } t \notin\left\{t_{0}, \ldots, t_{N}\right\} .
\end{aligned}
$$

- for any date $t$, there exists a positive random variable $c_{t}$ such that for any simple strategy $\theta$ with trading dates $t_{0}, \ldots t_{N}$,

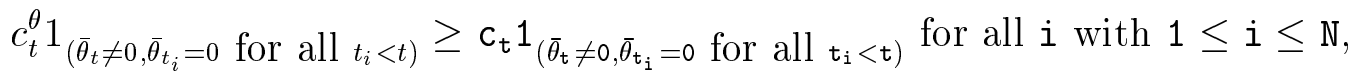

i.e., the first time real trading occurs, the fixed cost must be positive.

Or,

there exists a positive real number $\varepsilon$ such that for any simple strategy $\theta$ with trading dates $t_{0}, \ldots t_{N}$,

$$
\sum_{\left\{t_{i}\right\}} c_{t_{i}}^{\theta} \geq \varepsilon
$$

i.e., the cumulative transaction cost from the first to the last trading date must be greater than some positive constant.

- for all $t$, there exists a positive real number $C_{t}$ such that for any simple strategy $\theta$

$$
c_{t}^{\theta} \leq \mathrm{C}_{\mathrm{t}}
$$

i.e., the transaction cost is bounded at each date. This implies that for any simple strategy $\theta$ with trading dates $t_{0}, \ldots t_{N}$, the cumulative transaction $\operatorname{cost} \sum_{\left\{t_{i}\right\}} c_{t_{i}}^{\theta}$ is smaller than or equal to some constant (that depends on the strategy $\theta$ only, and not on the state of the world).

We could indifferently assume that for any strategy $\theta$ and any trading date $t$, the transaction cost at time $t$ is such that $\frac{c_{t}^{\lambda \theta}}{\lambda} \rightarrow_{\lambda \rightarrow \infty} 0$, which means that the transaction cost per unit of security traded goes to zero as the amount traded becomes arbitrarily large. 
Note that these conditions are consistent with a large class of transaction costs that can be identified in financial markets. They include fixed brokerage fees or brokerage arrangements where marginal fees go to zero beyond a given volume that is reset periodically (such arrangements are common in the industry), and fixed investment taxes to gain access to a market such as a foreign market. They also include operational and trade processing costs that typically exhibit strong economies of scale (especially if these tasks have been automated), and fixed costs incurred in setting up an office and obtaining access to price or other relevant information. Also, the opportunity cost of focusing on a market or of doing a specific trade can be viewed as a fixed cost.

In order to get some of our results, we shall need the following additional assumption $^{6}$ (that we shall mention each time it is needed):

Assumption $\mathbf{A}$ : There exists a real number $C$ such that for every strategy $\theta, \sum_{t \in \mathcal{T}} c_{t}^{\theta}<C$.

This means that, under Assumption A, the cumulative transaction costs of any trading strategy are assumed to be bounded by a constant. Note that this condition is automatically satisfied in a discrete time model with a finite or infinite number of states of the world (as long as transaction costs are bounded at each time), but a finite number of possible trading dates. It is also automatically satisfied in a model where there is a fixed cost to access a market such as a fixed investment tax, a fixed cost for setting up information technology or a trade processing department, or a fixed opportunity cost of looking at a market. It is also consistent with a situation where the fixed transaction costs consist in brokerage fees with a brokerage arrangement where transactions go free beyond a certain volume which is reset on a periodical basis (this type of arrangement is common in the industry).

Agents transfer wealth from all dates and events (for contingent wealth) to the terminal date using the traded securities, subject to paying the fixed transaction costs. In doing so they use self-financing strategies defined as follows. Let $i$ be a date in $F$ and let $B$ be an event in $F_{i}$ (in the remainder of the paper we shall always suppose that $P(B) \neq 0)$. We then have:

\footnotetext{
${ }^{6}$ For instance, we shall need Assumption A when we use the same definition of free lunch as in Kreps (1981). However, we shall also introduce an alternative definition of free lunch for which Assumption A is not required for any of our results.
} 
Definition 2.1 A self financing simple strategy from the date $i$ and the event $B$ is a strategy $\theta$ that is null before the date $i$ and outside the event $B=$ $\left\{\bar{\theta}_{i} \neq 0\right\}$, and such that there exist trading dates $t_{0}, \ldots, t_{N}$, with $i=t_{0} \leq \ldots \leq$ $t_{N}=T$, for which $\theta(t, \omega)$ is a.s. constant over each interval $\left[t_{k-1} ; t_{k}[\right.$ and satisfies

$$
\theta_{t_{k}} \cdot Z_{t_{k}}+c_{t_{k}}^{\theta} \leq \theta_{t_{k-1}} \cdot Z_{t_{k}} \text { for } k=1, \ldots, N \Leftrightarrow 1
$$

and

$$
\theta_{T} \cdot Z_{T}+c_{T}^{\theta}=\theta_{t_{N-1}} \cdot Z_{T}
$$

This means that a self-financing simple strategy does not require any additional investment beyond what is required at the initial date: purchases of securities as well as transaction costs after the initial date are financed by the sale of other securities. Let $S^{i, B}$ denote the set of such strategies. We also have:

Definition 2.2 A frictionless self financing simple strategy from the date $i$ and the event $B$ is a strategy $\theta$ that is null before the date $i$ and outside the event $B$ and such that there exist trading dates $t_{0}, \ldots, t_{N}$ with $i=t_{0} \leq \ldots \leq$ $t_{N}=T$ for which $\theta(t, \omega)$ is a.s. constant over each interval $\left[t_{k-1} ; t_{k}[\right.$ and satisfies $\theta_{t_{k}} \cdot Z_{t_{k}}=\theta_{t_{k-1}} \cdot Z_{t_{k}}$ a.s. $P$ for $k=1, \ldots, N$.

This means that a frictionless self financing simple strategy is a self financing simple strategy in an otherwise identical economy where there are no transaction costs. Let $W^{i, B}$ denote the set of such strategies.

\section{Arbitrage opportunities and free lunches}

\subsection{Arbitrage opportunities}

An arbitrage opportunity is a trading strategy that yields a positive gain in some circumstances without a countervailing threat of loss in any other circumstances. A free lunch is the possibility of getting arbitrarily close to an arbitrage opportunity. We shall define two concepts of arbitrage opportunities as follows :

Definition 3.1 1. An arbitrage opportunity with fixed costs $\left(A O_{1}\right)$ is a strategy $\theta$ such that there exist $(i, j)$ in $F, 0 \leq i \leq j \leq T$, an event 
$B$ in $F_{i}$, for which $\bar{\theta}$ is null after date $j, \theta$ belongs to $S^{i, B}, V_{i}^{\theta}+c_{i}^{\theta} \leq$ 0 on $B, V_{j}^{\theta} \geq 0$ and either $V_{i}^{\theta}+c_{i}^{\theta}$ or $V_{j}^{\theta}$ is different from 0 .

2. A frictionless strong arbitrage opportunity $\left(\mathrm{AO}_{2}\right)$ is a strategy $\theta$ such that there exist $(i, j)$ in $F, 0 \leq i \leq j \leq T$, an event $B$ in $F_{i}$, for which $\bar{\theta}$ is null after date $j, \theta$ belongs to $W^{i, B}, V_{i}^{\theta}<0$ on $B$ and $V_{j}^{\theta} \geq 0$.

This means that an $A O_{1}$ is a trading strategy that yields, in our model with fixed transaction costs, a positive gain in some circumstances without a threat of loss in other circumstances. An $\mathrm{AO}_{2}$ is a trading strategy that yields a positive gain at the starting date and event of the trading strategy without a countervailing threat of loss in other circumstances. We then have:

Proposition 3.1 1. There exists an $A O_{1}$ if and only if there exists a net gain arbitrage opportunity with fixed trading costs, i.e. a strategy $\theta$ such that there exist a date $i$ in $\mp$ and an event $B$ in $F_{i}$ for which $\theta$ belongs to $S^{i, B}$, and $\left[V_{T}^{\theta} \Leftrightarrow V_{i}^{\theta} \Leftrightarrow c_{i}^{\theta}\right] \geq 0, \neq 0$ on $B$.

2. There exists an $\mathrm{AO}_{2}$ if and only if there exists a frictionless $\varepsilon$-net gain arbitrage opportunity, i.e. a strategy $\theta$ such that there exist a date $i$ in $F$, an event $B$ in $F_{i}$ and a positive real number $\varepsilon$ for which $\theta$ belongs to $W^{i, B}$ and $V_{T}^{\theta} \Leftrightarrow V_{i}^{\theta} \geq \varepsilon$ on $B$.

3. There exists an $\mathrm{AO}_{1}$ if and only if there exists an $\mathrm{AO}_{2}$.

This means that the two notions of arbitrage opportunities that we have introduced are equivalent. Also, an arbitrage opportunity in our model with fixed transaction costs corresponds to the possibility of achieving a positive net gain. An arbitrage opportunity in the otherwise identical frictionless model corresponds to a net gain that is greater than some positive constant in all states of the world. It is hence clear that the set of arbitrage opportunities in our model with fixed transaction costs is strictly smaller than the set of arbitrage opportunities in the frictionless model, or equivalently that the assumption of no arbitrage in our model with fixed transaction costs is less stringent than in the frictionless model. 


\subsection{Free lunches}

As in Kreps (1981), we define a free lunch as the possibility of getting arbitrarily close to an arbitrage opportunity. More precisely, we have

Definition 3.2 1. A free lunch with fixed costs $\left(F L_{1}\right)$ is a sequence $\left(\theta^{n}\right)_{n \in N}$ of trading strategies such that there exist $i$ in $F, B$ in $F_{i}$, sequences $\left(x^{n}\right)_{n \in N}$ and $\left(\varepsilon_{i}^{n}\right)_{n \in N}$ of random variables belonging respectively to $L^{1}(\Omega, F, P)$ and $L^{1}\left(\Omega, F_{i}, P\right)$ and converging in $L^{1}(\Omega, F, P)$ respectively to $x \geq 0$ and $\varepsilon_{i} \geq 0$ on $B$ with $x+\varepsilon_{i} \neq 0$ for which for all $n$,

$$
\theta^{n} \text { is in } S^{i, B}, V_{i}^{\theta^{n}}+c_{i}^{\theta^{n}} \leq \Leftrightarrow \varepsilon_{i}^{n} \text { on } B \text { and } V_{T}^{\theta^{n}} \geq x^{n} \text {. }
$$

2. A frictionless strong free lunch $\left(F L_{2}\right)$ is a sequence $\left(\theta^{n}\right)_{n \in N}$ of trading strategies such that there exist $i$ in $F, B$ in $F_{i}$ and sequences $\left(x^{n}\right)_{n \in N}$ and $\left(r^{n}\right)_{n \in N}$ of random variables belonging respectively to $L^{1}(\Omega, F, P)$ and $L^{1}\left(\Omega, F_{i}, P\right)$ and converging in $L^{1}(\Omega, F, P)$ respectively to $x \geq 0$ and $r>0$ on $B$ for which for all $n$,

$$
\theta^{n} \text { is in } W^{i, B} \text { and satisfies } V_{i}^{\theta^{n}} \leq \Leftrightarrow r^{n} \text { and } V_{T}^{\theta^{n}} \geq x^{n} \text {. }
$$

3. An "asymptotic free lunch" $\left(\right.$ AsF L) is a sequence $\left(\theta^{n}\right)_{n \in N}$ of strategies such that there exist $i$ in $F, B$ in $F_{i}$, a sequence $\left(\lambda_{n}\right)_{n \geq 0}$ of positive real numbers and sequences $\left(x^{n}\right)_{n \in N}$ and $\left(\varepsilon_{i}^{n}\right)_{n \in N}$ of random variables belonging respectively to $L^{1}(\Omega, F, P)$ and $L^{1}\left(\Omega, F_{i}, P\right)$ and converging in $L^{1}(\Omega, F, P)$ respectively to $x \geq 0$ and $\varepsilon_{i}>0$ on $B$ for which for all $n$,

$$
\theta^{n} \text { is in } S^{i, B}, \frac{V_{i}^{\theta^{n}}+c_{i}^{\theta^{n}}}{\lambda_{n}} \leq \Leftrightarrow \varepsilon_{i}^{n} \text { on } B \text { and } \frac{V_{T}^{\theta^{n}}}{\lambda_{n}} \geq x^{n} \text {. }
$$

This means that a free lunch is a sequence of strategies with a payoff that converges to an arbitrage opportunity. A frictionless strong free lunch is a sequence of strategies with a payoff that converges to a frictionless strong arbitrage opportunity. An "asymptotic free lunch" is a sequence of strategies that are strong free lunches when renormalized by a sequence of scaling factors. We introduce this notion in order to avoid using Assumption A in our characterization Theorems in the next section.

Note that, as in the definition of arbitrage opportunities, we could replace the date $T$ with any date $j$, satifying $0 \leq i \leq j \leq T$ for which $\bar{\theta}^{n}$ is null after the date $j$. We then have 
Proposition 3.2 1. There exists a $F L_{1}$ if and only if there exists a net gain free lunch with fixed costs, i.e. a sequence $\left(\theta^{n}\right)_{n \in N}$ of strategies such that there exist $i$ in $F, B$ in $F_{i}$ and a sequence $\left(x^{n}\right)_{n \in N}$ of random variables belonging to $L^{1}(\Omega, F, P)$ and converging in $L^{1}(\Omega, F, P)$ to some $x \geq 0, \neq 0$ on $B$ for which for all $n, \theta^{n}$ is in $S^{i, B}$ and $V_{T}^{\theta^{n}} \Leftrightarrow$ $\left(V_{i}^{\theta^{n}}+c_{i}^{\theta^{n}}\right) \geq x^{n}$.

2. There exists a $F L_{2}$ if and only if there exists a frictionless $\varepsilon$-net gain free lunch, i.e., a sequence $\left(\theta^{n}\right)_{n \in N}$ of strategies such that there exist $i$ in F, $B$ in $F_{i}$, a positive real number $\varepsilon$ and a sequence $\left(x^{n}\right)_{n \in N}$ of random variables belonging to $L^{1}(\Omega, F, P)$ and converging to some $x \geq \varepsilon$ on $B$ for which for all $n, \theta^{n}$ is in $W^{i, B}$ and satisfies $V_{T}^{\theta^{n}} \Leftrightarrow V_{i}^{\theta^{n}} \geq x^{n}$.

This means that a free lunch corresponds to a sequence of trading strategies with a payoff that converges to a positive net gain. Similarly a frictionless strong free lunch corresponds to a sequence of trading strategies with a payoff that converges to a net gain that is strictly positive in all states of the world. We then have the following characterization of the absence of frictionless strong free lunches:

Corollary 3.1 Let $K^{i, B}=\left\{V_{T}^{\theta} \Leftrightarrow V_{i}^{\theta} ; \theta \in W^{i, B}\right\} \subset L^{1}(\Omega, F, P)$, the set of possible gains from date $i$ and event $B$ in the frictionless model, and $C^{i, B}=$ $\overline{K^{i, B} \Leftrightarrow L_{+}^{1}}$, where the closure is taken in $L^{1}$. Let

$$
A^{B}=\left\{f \in L_{+}^{1}, \exists \varepsilon>0 \text { such that } f \geq \varepsilon \text { on } B\right\} .
$$

The assumption of no frictionless strong free lunch $\left(N F L_{2}\right)$ is equivalent to the condition that for all $i$ in $F$ and $B$ in $F_{i}$, the two convex sets $C^{i, B}$ and $A^{B}$ have an empty intersection.

We also have

Lemma 3.1 1. The absence of frictionless strong free lunch $\left(\mathrm{NFL}_{2}\right) \mathrm{im}$ plies the absence of free lunch in our model with fixed trading costs $\left(N F L_{1}\right)$.

2. Under Assumption A, the absence of free lunch in our model with fixed costs $\left(N F L_{1}\right)$ and the absence of frictionless strong free lunch $\left(N F L_{2}\right)$ are equivalent. 
3. The absence of "asymptotic free lunch" $(N A s F L)$ in our model with fixed trading costs and the absence of frictionless strong free lunch $\left(N F L_{2}\right)$ are equivalent.

It is easy to see that the absence of frictionless strong free lunch implies the absence of free lunch with fixed trading costs. But, unlike for arbitrage opportunities, the converse is not necessarily true. Indeed, although the number of trading dates for each trading strategy $\theta^{n}$ is finite, it can be arbitrarily large, and therefore so can the cumulative trading costs. Hence the need to bound the total trading costs of any simple strategy as in Assumption A or to consider the notion of "asymptotic free lunch". In both cases we obtain the equivalence between the absence of strong frictionless free lunches and the absence of free lunches in our model with fixed trading costs.

\subsection{Absolutely continuous martingale measures}

With the notations of corollary ??, it is easy to see, using the definition of the set of self financing simple trading strategies in the frictionless model $W^{i, B}$ and the fact that $Z_{t}^{0}=1$, that $K^{i, B}=\left\{V_{T}^{\theta} \Leftrightarrow V_{i}^{\theta} ; \theta \in W^{i, B}\right\}$, the set of possible gains from date $i$ and event $B$ in the frictionless model, is a vector space and that it is equal to $K^{i, B}=\operatorname{Lin}\left\{\theta_{s} \cdot\left(\bar{Z}_{t} \Leftrightarrow \bar{Z}_{s}\right) ; \theta_{s} \in P^{s, B}, i \leq s \leq t\right\}$, where for all $s \geq i, \bar{Z}_{s}=\left(Z_{s}^{1}, \ldots, Z_{s}^{n}\right)$ and where $P^{s, B}$ denotes the set of $n$-dimensional random variables $\theta_{s}=\left(\theta_{s}^{1}, \ldots, \theta_{s}^{n}\right)$ that are $F_{s}$-measurable, null outside $B$ and before $i$ and such that $\theta_{s} \cdot \bar{Z}_{s}$ is in $L^{1}\left(\Omega, F_{s}, P\right)$.

The use of corollary ?? and of a separation theorem will now enable us to obtain our main result: the characterization of the absence of frictionless strong free lunches in terms of absolutely continuous martingale mesures.

Theorem 3.1 There exists no frictionless strong free lunch if and only if for all $i$ in $F$ and all $B$ in $F_{i}$, there exists an absolutely continuous probability measure $P^{i, B}$ defined on $(\Omega, F)$, with bounded density, such that $P^{i, B}(B)=1$ and $E^{P^{i, B}}\left[Z_{t} \mid F_{s}\right]=Z_{s}$ for all $(s, t)$ such that $i \leq s \leq t$.

We then obtain the Fundamental Theorem of Asset Pricing for securities markets models with fixed trading costs.

Theorem 3.2 The following are equivalent: 
1. There exists no "asymptotic free lunch" in our model with fixed trading costs.

2. There exists a family of absolutely continuous martingale measures: for all $i$ in $F$ and for all $B$ in $F_{i}$, there exists an absolutely continuous probability measure with bounded density $P^{i, B}$ defined on $(\Omega, F)$ such that $P^{i, B}(B)=1$ and satisfying

$$
E^{P^{i, B}}\left[Z_{t} \mid F_{s}\right]=Z_{s} \text { for all }(s, t) \text { with } i \leq s \leq t .
$$

3. There exists a family of nonnegative state price densities: for all $i$ in F and for all $B$ in $F_{i}$, there exists a bounded random variable $g^{i, B}$ in $L^{\infty}(\Omega, F, P)$ with $g^{i, B} \geq 0, \neq 0$ on $B$ and such that for all $(s, t)$ with $i \leq s \leq t$

$$
E\left[g^{i, B} Z_{t} 1_{A \cap B}\right]=E\left[g^{i, B} Z_{s} 1_{A \cap B}\right] \text { for all } A \text { in } F_{s} .
$$

4. There exists a family of weakly positive ${ }^{7}$ continuous linear operators: let $R^{i, B}$ denote the set of random variables null outside $B$ and belonging to $L^{1}\left(\Omega, F_{i}, P\right)$. For all $i$ in $F$, for all $B$ in $F_{i}$, there exists a weakly positive continuous linear operator $\pi^{i, B}$ defined on $R^{T, B}$ and taking values in $R^{i, B}$, such that there exists an event $A$ in $F_{i}$ with $A \subset B$ and $P(A) \neq 0$ for which

$$
\pi^{i, B}\left(V_{T}^{\theta}\right)=V_{i}^{\theta} \text { on } A \text {, for all } \theta \text { in } W^{i, B} .
$$

Under Assumption A, these statements are all equivalent to:

5. There exists no free lunch in our model with fixed trading costs.

This means that the absence of free lunches in our model with fixed trading costs (or equivalently the absence of free lunches in the otherwise identical frictionless model) is equivalent to the existence of a family of absolutely continuous martingale probability measures: absolutely continuous martingale measures conditional on any possible future event. Note the difference with the frictionless case where the absence of free lunches (a weaker condition than the absence of free lunches in the model with fixed trading costs) is

\footnotetext{
${ }^{7}$ Let $X$ denote the set of random variables on $(\Omega, F, P)$. A functional $p$ defined on $X$ is said to be weakly positive if for all $x$ in $X$ such that $P(x \geq 0)=1$, we have $p(x) \geq 0$.
} 
equivalent to the existence of an equivalent martingale probability measures (a stronger condition since a family of absolutely continuous martingale measures can be derived from any equivalent martingale measure) as shown in Harrison and Kreps (1979).

We can also obtain the slightly more general results in the spirit of Yan's (1980) theorems ( also see Stricker (1990), among others, for an application of Yan's theorem).

Theorem 3.3 Let $K$ be a convex set in $L^{1}(\Omega, F, P)$ containing 0 . The following conditions are equivalent :

1. For all $\eta$ in $L^{1}$ such that $\eta>0$, there exists a positive real number $c$ for which cn is not in $\overline{K \Leftrightarrow L_{+}^{1}}$.

2. There exists a positive real number $c$ such that $c 1_{\Omega}$ is not in $\overline{K \Leftrightarrow L_{+}^{1}}$.

3. There exists a random variable $Z$ in $L^{\infty}(\Omega, F, P)$ satisfying $Z \geq 0, \neq 0$ and $\sup _{\zeta \in K} E[Z \zeta]<\infty$

We also have

Corollary 3.2 Let $K$ denote $K^{0, \Omega}$ with

$K^{i, B}=\left\{V_{T}^{\theta} \Leftrightarrow V_{i}^{\theta}, \theta \in W^{i, B}\right\}=\operatorname{Lin}\left\{\theta_{s} \cdot\left(\bar{Z}_{t} \Leftrightarrow \bar{Z}_{s}\right) ; \theta_{s}\right.$ is in $\left.P^{s, B}, t \geq s \geq i\right\}$

where for all $s \geq i, \bar{Z}_{s}=\left(Z_{s}^{1}, \ldots, Z_{s}^{n}\right)$ and $P^{s, B}$ denotes the set of $n$ dimensional random variables $\theta_{s}=\left(\theta_{s}^{1}, \ldots, \theta_{s}^{n}\right)$ that are $F_{s}$-measurable, null outside $B$ and before $i$, and such that $\theta_{s} \cdot Z_{s}$ is in $L^{1}\left(\Omega, F_{s}, P\right)$. Also, let $A^{B}$ denote $\left\{f \in L_{+}^{1}, \exists \varepsilon>0\right.$ such that $f \geq \varepsilon$ on $\left.B\right\}$. The following conditions are equivalent:

1. The intersection $A^{\Omega} \cap \overline{K \Leftrightarrow L_{+}^{1}}$ is empty.

2. The random variable $1_{\Omega}$ does not belong to $\overline{K \Leftrightarrow L_{+}^{1}}$.

3. There exists an absolutely continuous martingale measure for $Z$. 
This concludes our characterization of processes that admit an absolutely continuous martingale measure - which relates to the Theorem of Asset Pricing in securities markets models with fixed trading costs (note that the implications 2) $\Rightarrow 1$ ) in Theorems ?? and Corollary ?? are quite general and can be useful in other contexts as well). The characterization of processes that admit an equivalent martingale measure (or the Fundamental Theorem of Asset Pricing in frictionless securities markets models) can be found in Harrison and Kreps (1979), Yan (1980), Kreps (1981), Duffie and Huang (1986), Stricker (1990) or Delbaen and Schachermayer (1994 and 1998), as well as Back and Pliska (1988) and Dalang et al. (1980) for the discrete time case.

We shall now exhibit an example of a process that admits a family of absolutely continuous martingale probability measures but does not admit any equivalent martingale probability measure.

Example (Delbaen and Schachermayer (1994)): Let $W$ be a standard Wiener process, with its natural filtration $\left(G_{t}\right)_{0 \leq t \leq 1}$. We define a local martingale of exponential type by:

$$
\begin{aligned}
& L_{t}=\exp \left(\Leftrightarrow(f \cdot W)_{t} \Leftrightarrow \frac{1}{2}\left(\int_{0}^{t} f^{2}(u) d u\right)\right) \text { if } t<1, \text { and } \\
& L_{1}=0,
\end{aligned}
$$

where $f(t)=\frac{1}{\sqrt{1-t}}$. We define the stopping time $T$ by $T=\inf \left\{t, L_{t} \geq 2\right\}$. We then define the price process $S_{t}$ by:

$$
d S_{t}=d W_{t}+\frac{1}{\sqrt{1-t}} d t \text { if } t \leq T, \text { and }
$$$$
d S_{t}=0 \text { if } t \geq T \text {, }
$$

and the filtration $\left(F_{t}\right)_{0 \leq t \leq 1}=\left(G_{\min (t, T)}\right)_{0 \leq t \leq 1}$.

According to Delbaen and Schachermayer (1994), there exists a unique probability measure $Q$ that is absolutely continuous with respect to $P$ and makes the process $S$ a martingale. It is given by $d Q=L_{T} d P$. Since $P\left[L_{T}=\right.$ $0]>0, \mathrm{Q}$ is not equivalent to $P$. Moreover, for all $t<1$, the measures $Q$ and $P$ are equivalent on $F_{t}$ since the density $L_{T}$ is positive. It is now easy to see that for any date $t$ and for all event $B$ at that date, there exists a probability measure $Q^{t, B}$ given by $d Q^{t, B}=\frac{L_{T} 1_{B}}{E\left[L_{T} 1_{B}\right]} d P$ such that $Q^{t, B}(B)=1$ and $E^{Q^{t, B}}\left[S_{v} \mid F_{u}\right]=S_{u}$ for all $(u, v)$ with $t \leq u \leq v$. 


\section{Pricing and viability with fixed costs}

\subsection{Admissible pricing rules}

A contingent claim $B$ to consumption at the terminal date $T$ is a random variable belonging to $L^{1}(\Omega, F, P)$. A contingent claim $B$ is said to be attainable (in the model without fixed cost) if there exists some frictionless self financing strategy $\theta$ in $W^{0, \Omega}$ such that $V_{T}^{\theta}=B$. Note that the set $M$ of all attainable contingent claims is a linear space. We shall now define and characterize pricing rules $p(B)$ on $M$ that are admissible.

Definition 4.1 An admissible pricing rule on $M$ is a functional $p$ defined on $M$, such that

1. $p$ induces no arbitrage, i.e., it is not possible to find strategies $\theta_{1}, \ldots, \theta_{n}$ in $W^{0, \Omega}$, for which $\sum_{i=1}^{n} p\left(V_{T}^{\theta_{i}}\right) \leq 0, \sum_{i=1}^{n} V_{T}^{\theta_{i}} \geq 0$ and one of the two is nonnull.

2. $p(B) \leq \pi_{s}(B)$, where $\pi_{s}(B):=\inf \left\{V_{0}^{\theta}+c_{0}^{\theta}, \theta \in S^{0, \Omega}, V_{T}^{\theta} \geq B\right\}$.

Part 1 is the usual no-arbitrage condition. Part 2 says that an admissible price for the contingent claim $B$ must be smaller than its superreplication price: if it is possible to obtain a payoff at least equal to $B$ at a cost $\pi_{s}(B)$, then no rational agent (who prefers more to less) will accept to pay more than $\pi_{s}(B)$ for the contingent claim $B$. Note that since $B$ is attainable by a frictionless self financing strategy, and since the total trading costs incurred by any strategy are bounded, there always exists at least a self financing (inclusive of transaction costs) strategy dominating $B$, i.e. $B$ is also attainable in our model with fixed trading costs.

The following Proposition characterizes the admissible pricing rules on $M$ through the use of the absolutely continuous martingale measures obtained in Theorem ??.

Proposition 4.1 Under Assumption $A$ and the assumption of $N F L_{1}$, or under the assumption of NAsFL, any admissible pricing rule $p$ on $M$ can be written as

$$
p(B)=E^{P^{*}}[B]+c(B) \quad \text { for all } B \text { in } M
$$

where $P^{*}$ is any absolutely continuous martingale measure and $\frac{c(\lambda B)}{\lambda} \rightarrow_{\lambda \rightarrow \infty}$ 0 . 
This means that if $B=V_{T}^{\theta}$ then $p(B)=V_{0}^{\theta}+c(B)$ since $E^{P^{*}}\left(V_{T}^{\theta}\right)=V_{0}^{\theta}$ for any absolutely continuous martingale measure $P^{*}$. Moreover, if $p(\lambda x) \leq$ $\lambda[p(x)]$ for any real number $\lambda$ large enough, then the fixed cost $c$ is nonnegative. And if there exists $\varepsilon>0$, such that for any $\lambda$ large enough, $p(\lambda x) \leq \lambda[p(x) \Leftrightarrow \varepsilon]$, then the fixed $\operatorname{cost} c$ is greater than or equal to this positive constant $\varepsilon$. Notice that under Assumption A, i.e. if the cumulative fixed costs incurred by any strategy are bounded by a positive real number $C$, then $c(B):=p(B) \Leftrightarrow E^{P^{*}}(B) \leq \pi_{s}(B) \Leftrightarrow E^{P^{*}}(B) \leq C$, for any absolutely continuous martingale measure $P^{*}$. Also, Proposition ?? implies that $\frac{p(\lambda B)}{\lambda} \rightarrow_{\lambda \rightarrow \infty} E^{P^{*}}[B]$ for any attainable contingent claim $B$, where $P^{*}$ is any absolutely continuous martingale measure. This means that the unit price of any attainable contingent claim $B$ is equal to $E^{P^{*}}[B]$ in the limit of large quantities.

As usual, we say that the market is complete in the frictionless model if any contingent claim is attainable. If the market is complete, there exists a unique admissible pricing rule. However, in incomplete markets (i.e., if there are some non attainable contingent claims), even in a frictionless model there is no universal pricing concept. We can only find arbitrage bounds and the pricing rules are sublinear ${ }^{8}$ lower semicontinuous functionals (see Jouini and Kallal (1995a and 1999)). By analogy with the case of attainable contingent claims, we define an admissible pricing rule on the set of contingent claims in the following way.

Definition 4.2 A pricing rule on $L^{1}(\Omega, F, P)$ is admissible if it is of the form $p(B)=\pi(B)+c(B)$ for all $B$ in $L^{1}(\Omega, F, P)$, where

1. $\pi$ is a sublinear lower semicontinuous functional and $c$ is such that $\frac{c(\lambda B)}{\lambda} \rightarrow_{\lambda \rightarrow \infty} 0$.

2. $p(B) \leq \pi_{s}(B)$, where $\pi_{s}(B):=\inf \left\{V_{0}^{\theta}+c_{0}^{\theta}, \theta \in S^{0, \Omega}, V_{T}^{\theta} \geq B\right\}$

We then obtain the following characterization of the admissible pricing rules.

\footnotetext{
${ }^{8} \mathrm{~A}$ functional $\pi$ is sublinear if $\pi(\lambda x)=\lambda \pi(x)$ and $\hat{e} \pi(x+y) \leq \pi(x)+\pi(y)$ for all contingent claims $x, y$ and nonnegative real numbers $\lambda$.
} 
Proposition 4.2 Under Assumption $A$ and the assumption of $N F L_{1}$, or under the assumption of NAsFL, any admissible pricing rule $p$ on $L^{1}(\Omega, F, P)$ can be written as

$$
p(B)=\sup _{P^{*} \in K} E^{P^{*}}[B]+c(B) \quad \text { for all } B \text { in } M
$$

where $K$ denotes a convex subset of the set of all absolutely continuous martingale measures, and $c$ is the fixed cost given in Definition 6.

This means that any admissible sublinear lower semicontinuous functional $\pi$ can be written as the supremum of a subset of all continuous linear functionals $\tilde{l}$, which lie below $\pi$, are weakly positive and such that $\tilde{l}\left(V_{T}^{\theta}\right)=V_{0}^{\theta}$ for all $\theta$ in $W^{0, \Omega}$. It also means that $\frac{p(\lambda B)}{\lambda} \rightarrow_{\lambda \rightarrow \infty} \sup _{P^{*} \in K} E^{P^{*}}[B]$ for any contingent claim $B$, where $K$ is a convex subset of the set of absolutely continuous martingale measures. This means that the unit price of any attainable contingent claim $B$ must belong to an interval $\left[\Leftrightarrow \inf _{P^{*} \in K} E^{P^{*}}[\Leftrightarrow B], \sup _{P^{*} \in K} E^{P^{*}}[B]\right]$ in the limit of large quantities.

Note that since the absence of free lunch in our model with fixed trading costs is weaker than the absence of free lunch in a frictionless model, these theorems enable us to price contingent claims in a wider class of models. We shall now turn to the study of the viability of such admissible pricing rules.

\subsection{Viability}

Agents are assumed to be characterized by their preferences on the space of net trades $R \times X$ where $X=L^{1}(\Omega, F, P)$. A pair $(r, x)$ represents $r$ units of consumption today and $x$ units of consumption tomorrow. Preferences are modeled by complete and transitive binary relations $\succeq$ on $R \times X$. In the usual fashion, $\succ$ denotes the strict preference defined from $\succeq$. We also make Assumption P: Preferences are assumed to satisfy the following three requirements:

1. For all $(r, x) \in R \times X$, the set $\left\{\left(r^{\prime}, x^{\prime}\right) \in R \times X:\left(r^{\prime}, x^{\prime}\right) \succeq(r, x)\right\}$ is convex.

2. For all $(r, x) \in R \times X$, the set $\left\{\left(r^{\prime}, x^{\prime}\right) \in R \times X:\left(r^{\prime}, x^{\prime}\right) \succeq(r, x)\right\}$ as well as the set $\left\{\left(r^{\prime}, x^{\prime}\right) \in R \times X:(r, x) \succeq\left(r^{\prime}, x^{\prime}\right)\right\}$ are closed. 
3. For all $(r, x) \in R \times X, r^{\prime}>0$ and $x^{\prime} \in L_{+}^{1}$ such that there exists a real number $\varepsilon>0$ with $x^{\prime} \geq \varepsilon,\left(r+r^{\prime}, x\right) \succ(r, x)$ and $\left(r, x+x^{\prime}\right) \succ(r, x)$.

The class of such preferences is denoted by $A$. Part 1 says that agents are risk averse. Part 2 says that their preferences are continuous. Part 3 says that agents prefer more to less.

A price system $(M, p)$ is a subspace $M$ of $X$ and a linear functional $p$ on $M$. In the economy associated to this price system, agents can buy and sell any contingent claim $m \in M$ at a price $p(m)+c(m)$ in terms of date 0 consumption where $c(m)$ is a bounded nonnegative fixed trading cost satisfying $c(0)=0$ and $c(m)>0$ if $m \neq 0$.

Definition 4.3 A price system $(M, p)$ is said to be viable if there exist some binary relation $\succeq$ satisfying Assumption $P$ and $\left(r^{*}, m^{*}\right)$ in $R \times M$ such that $c\left(m^{*}\right)+r^{*}+p\left(m^{*}\right) \leq 0$ and

$$
\left(r^{*}, m^{*}\right) \succeq(r, m)
$$

for all $(r, m)$ in $R \times M$ such that $c(m)+r+p(m) \leq 0$.

This definition is analogous to the definition in Harrison and Kreps (1979) and Kreps (1981). It means that a price system is viable if there is some agent with preferences satisfying Assumption $P$ who can find an optimal net trade subject to his budget constraint. Note that if we assume that the fixed cost function $c$ is subadditive, i.e. $c\left(m_{1}+m_{2}\right) \leq c\left(m_{1}\right)+c\left(m_{2}\right)$ for all $m_{1}, m_{2} \in M$, a natural assumption to make about fixed costs, then a price system is viable if and only if there are some agents with preferences satisfying Assumption $P$ for whom $(0,0)$ is an optimal trade, ${ }^{9}$ i.e. who are happy with their initial endowment. This means that a price system is viable if and only if it is compatible with economic equilibrium.

\footnotetext{
${ }^{9}$ Indeed, suppose that there exists an agent with preferences $\succeq$ satisfying Assumption $P$ and such that $\left(r^{*}, m^{*}\right)$ is an optimal net trade (i.e. $c\left(m^{*}\right)+r^{*}+p\left(m^{*}\right) \leq 0$ and $\left(r^{*}, m^{*}\right) \succeq(r, m)$ for all $\hat{e}(r, m)$ in $R \times M$ such that $\left.c(m)+r+p(m) \leq 0\right)$. Define the preferences $\tilde{\succeq}$ by $\left(r_{1}, m_{1}\right) \check{\succeq}\left(r_{2}, m_{2}\right)$ if $\left(r_{1}+r^{*}, m_{1}+m^{*}\right) \tilde{\succeq}\left(r_{2}+r^{*}, m_{2}+m^{*}\right)$. They satisfy Assumption $P$. Also note that $c(0)+0+p(0)=0$. Now suppose that $c(\tilde{m})+\tilde{r}+$ $p(\tilde{m}) \leq 0$ and $(\tilde{r}, \tilde{m}) \tilde{\succ}(0,0)$, i.e. $\left(\tilde{r}+r^{*}, \tilde{m}+m^{*}\right) \succ\left(r^{*}, m^{*}\right)$. We have $c\left(\tilde{m}+m^{*}\right)+\tilde{r}+$ $r^{*}+p\left(\tilde{m}+m^{*}\right)=\left[c\left(\tilde{m}+m^{*}\right)-c(\tilde{m})-c\left(m^{*}\right)\right]+c(\tilde{m})+\tilde{r}+p(\tilde{m})+c\left(m^{*}\right)+r^{*}+p\left(m^{*}\right) \leq$ $c\left(\tilde{m}+m^{*}\right)-c(\tilde{m})-c\left(m^{*}\right) \leq 0$ by subadditivity of the fixed cost functional $\hat{e} c$.
} 
Definition 4.4 A free lunch for a price system $(M, p)$ is a sequence $\left(m^{n}\right)_{n \in N}$ in $M$, such that there exist sequences $\left(r^{n}\right)_{n \in N},\left(x^{n}\right)_{n \in N}$ in $L^{1}(\Omega, F, P)$ converging respectively to $r \geq 0$ and $x \geq 0$ with $r+x \neq 0$, for which for all $n$ in $N$

$$
m^{n} \geq x^{n} \text { and } c\left(m^{n}\right)+r^{n}+p\left(m^{n}\right) \leq 0 .
$$

We shall now consider the case where $M=\left\{V_{T}^{\theta} ; \theta\right.$ in $\left.W^{0, \Omega}\right\}$, the set of attainable contingent claims in the frictionless economy, and where the pricing rule is the linear functional $p$ defined on $M$ by $p\left(V_{T}^{\theta}\right)=V_{0}^{\theta}$ for all $\theta$ in $W^{0, \Omega}$. As we have seen in Proposition ??, if we want a price system $(M, \pi)$ to be compatible with the assumption of no arbitrage - which must be the case for viable price systems as well as for price systems that admit no free lunch - then we must have $\pi=p$. We shall now investigate the converse, i.e. the conditions under which this price system is a viable one and the conditions under which it admits no free lunch. But first let us have:

Definition 4.5 A free lunch from time 0 in the frictionless securities market model is a sequence $\left(\theta^{n}\right)_{n \in N}$ of simple strategies such that there exist sequences $\left(\tilde{x}^{n}\right)_{n \in N}$ of random variables belonging to $L^{1}(\Omega, F, P)$ and $\left(\tilde{r}^{n}\right)_{n \in N}$ in $R^{N}$ converging respectively to $x \geq 0$ in $L^{1}(\Omega, F, P)$ and $r>0$ in $R$ for which for all $n$,

$$
\theta^{n} \text { is in } W^{0, \Omega}, \quad V_{0}^{\theta^{n}} \leq \Leftrightarrow \tilde{r}^{n} \text { and } V_{T}^{\theta^{n}} \geq \tilde{x}^{n} .
$$

We then have

Theorem 4.1 The following conditions are equivalent:

1. $(M, p)$ is viable.

2. $(M, p)$ admits no free lunch.

3. There exists a weakly positive continuous linear functional $\pi$ on $L^{1}(\Omega, F, P)$ such that $\left.\pi\right|_{M}=p$ and such that for all $f$ in $A=\left\{f \in L^{1}, \exists \varepsilon>0\right.$ such that $f \geq \varepsilon\}$, we have $\pi(f)>0$.

4. There is no free lunch from time 0. 
For each date $i$ and each event $B$ in $F_{i}$, we shall define a price system $\left(M^{i, B}, p^{i, B}\right)$ where $M^{i, B}$ is a subspace of $X$ and $p^{i, B}$ a linear functional on $M^{i, B}$. The interpretation is that in this economy, at that date $i$ and in that event $B$, agents are able to buy and sell some contingent claims $m$ in $M^{i, B}$ at a cost $p^{i, B}(m)+c_{i}(m)$ in date $i$, event $B$ consumption. We consider $M^{i, B}=\left\{V_{T}^{\theta} ; \theta\right.$ in $\left.W^{i, B}\right\}$ and $p^{i, B}$ defined on $M^{i, B}$ by $p^{i, B}\left(V_{T}^{\theta}\right)=V_{i}^{\theta}$ and we obtain

Theorem 4.2 The following conditions are equivalent:

1. For all $i$ in $F$, for all $B$ in $F_{i},\left(M^{i, B}, p^{i, B}\right)$ is viable.

2. For all $i$ in $F$, for all $B$ in $F_{i},\left(M^{i, B}, p^{i, B}\right)$ admits no free lunch.

3. There is no free lunch in our securities markets model with fixed trading costs.

Therefore, the price system we have considered is viable and admits no free lunch if and only if there is no free lunch in our model with fixed trading costs.

\section{Conclusion}

In this paper, we have shown that a securities markets model with fixed trading costs admits no free lunch if and only if there exists a family of absolutely continuous probability measures for which the normalized (by a numeraire) price processes are martingales, conditional on any possible future event. The main difference with the frictionless case is that the martingale measures only need to be absolutely continuous instead of equivalent (but we need a whole family of martingale measures). Since the absence of arbitrage opportunity or free lunch is a weaker condition in the presence of fixed trading costs than in the frictionless case, this result will allow future research to consider a wider class of models. The transaction costs are assumed to be fixed in the sense that they are bounded (regardless of the transaction size). This is compatible with fixed brokerage fees, brokerage arrangements where marginal fees go to zero beyond a given volume (a common arrangement in the industry), fixed investment taxes to gain access to a market, operational 
and processing costs, fixed costs involved in setting up an office and information technology, and the opportunity cost of looking at a market or of doing a specific trade. We also show that the only arbitrage free pricing rules on the set of attainable contingent claims are those that are equal to the sum of an expected value with respect to any absolutely continuous martingale measure and of a bounded fixed cost functional. Moreover, these pricing rules are the only ones to be viable as models of economic equilibrium, i.e. such that there exist some rational agents who are happy with their initial endowment - and hence for whom supply is equal to demand. 


\section{Appendix}

Proof of Proposition ??, We will write EAO for existence of an arbitrage opportunity and $N A O$ for no arbitrage opportunity. We will denote a net gain arbitrage opportunity with fixed costs by $\mathrm{AO}_{3}$ and a frictionless $\varepsilon$ net gain arbitrage opportunity by $A O_{4}$. We shall prove that the four notions of $N A O$ are equivalent. We first treat the case where the fixed costs do not depend upon the strategy.

1. $N A O_{3} \Leftrightarrow N A O_{1}: E A O_{1} \Rightarrow E A O_{3}$ is immediate. $E A O_{3} \Rightarrow E A O_{1}$ : we consider the strategy $\tilde{\theta}$ null before $i$ and outside $B$ such that for all $t \geq i$,

$$
\tilde{\theta}_{t}^{0}=\theta_{t}^{0}+\left(\Leftrightarrow c_{i} \Leftrightarrow V_{i}^{\theta}\right) \text { on } B \text { and } \tilde{\theta}_{t}^{k}=\theta_{t}^{k} \text { for all } k \neq 0 .
$$

It is easy to check that $\tilde{\theta}$ is in $S^{i, B}, V_{i}^{\tilde{\theta}}+c_{i}=0$ and $V_{T}^{\tilde{\theta}} \geq 0, \neq 0$ on $B$.

2. $N A O_{2} \Leftrightarrow N A O_{1}: E A O_{1} \Rightarrow E A O_{2}$ : we consider the strategy $\tilde{\theta}$ null before $i$ and outside $B$ such that

$$
\begin{aligned}
\tilde{\theta}_{i} & =\theta_{i} \text { and for all } t>i \\
\tilde{\theta}_{t}^{0} & =\theta_{t}^{0} \Leftrightarrow \sum_{j=i+1}^{t}\left(\theta_{j} \Leftrightarrow \theta_{j-1}\right) \cdot Z_{j} \text { on } B \text { and } \\
\tilde{\theta}_{t}^{k} & =\theta_{t}^{k} \text { for all } k \neq 0 .
\end{aligned}
$$

Then $\tilde{\theta}$ is in $W^{i, B}, V_{T}^{\tilde{\theta}} \geq 0$ and as $c_{i}>0$, we have $V_{i}^{\tilde{\theta}}<0$ on $B$. $E A O_{2} \Rightarrow E A O_{1}$ : notice that, by considering some $B^{\prime} \subset B$, one can replace the condition $V_{i}^{\theta}<0$ on $B$ by either the condition " $V_{i}^{\theta} \leq 0, \neq 0$ on $B$ " or by the condition "there exists a positive real number $\varepsilon$ such that $V_{i}^{\theta} \leq \Leftrightarrow \varepsilon$ on $B$ " because $V_{i}^{\theta}$ is $F_{i}$ emeasurable. So there exists $\lambda \geq$ 1 satisfying $\lambda V_{i}^{\theta} \leq \Leftrightarrow C$ where $C=\sum_{k=i}^{T} C_{k}$ and $C_{k}=\sup _{\omega \in B} c_{k}(\omega)$. We consider the strategy $\tilde{\theta}$ null before $i$ and outside $B$ such that for all $t \geq i$

$$
\begin{aligned}
\tilde{\theta}_{t}^{0} & =\lambda \theta_{t}^{0}+C \Leftrightarrow \sum_{j=i}^{t} c_{j} \text { and } \\
\tilde{\theta}_{t}^{k} & =\lambda \theta_{t}^{k} \text { for all } k \neq 0 .
\end{aligned}
$$

Then $\tilde{\theta}$ is in $S^{i, B}$ and satisfies $V_{i}^{\tilde{\theta}}+c_{i}=\lambda V_{i}^{\theta}+C \leq 0$ on $B, V_{T}^{\tilde{\theta}} \geq 0$. 
3. $N A O_{2} \Leftrightarrow N A O_{4}: E A O_{2} \Rightarrow E A O_{4}$ is easy with the technical remark made for the proof of $E A O_{2} \Rightarrow E A O_{1}$. $E A O_{4} \Rightarrow E A O_{2}$ : we consider the strategy $\tilde{\theta}$ null before $i$ and outside $B$ and such that for all $t \geq i$

$$
\begin{aligned}
\tilde{\theta}_{t}^{0} & =\theta_{t}^{0} \Leftrightarrow V_{i}^{\theta} \Leftrightarrow \varepsilon / 2 \text { on } B \text { and } \\
\tilde{\theta}_{t}^{k} & =\theta_{t}^{k} \text { for all } k \neq 0 .
\end{aligned}
$$

Then $\tilde{\theta}$ is in $W^{i, B}$ and satisfies $V_{i}^{\tilde{\theta}}=\Leftrightarrow(\varepsilon / 2)<0$ on $B$. We have $V_{T}^{\tilde{\theta}}=0$ outside $B$, and $V_{T}^{\tilde{\theta}} 1_{B}=\left(V_{T}^{\tilde{\theta}} \Leftrightarrow V_{i}^{\tilde{\theta}}\right) 1_{B}+V_{i}^{\tilde{\theta}} 1_{B}=\left(V_{T}^{\theta} \Leftrightarrow V_{i}^{\theta}\right) 1_{B} \Leftrightarrow(\varepsilon / 2) 1_{B}$ so $V_{T}^{\tilde{\theta}} \geq \varepsilon / 2$ on $B$ and $V_{T}^{\tilde{\theta}} \geq 0$.

If the costs depend upon the strategy, then $E A O_{1} \Rightarrow E A O_{3}$ is immediate. For $E A 0_{3} \Rightarrow E A O_{4}$, we easily get the existence of a strategy $\theta \in W^{i, B}$, $V_{T}^{\theta} \Leftrightarrow V_{i}^{\theta} \geq \mathrm{c}_{\mathrm{i}}$ on $B$. Then there exists $a>0$, such that for $B^{\prime} \equiv\left\{\mathrm{c}_{\mathrm{i}}>\mathrm{a}\right\}$, $P\left(B^{\prime}\right)>0$ and $\theta^{\prime} \equiv \theta 1_{B^{\prime}}$ is an $A O_{4}$. The proof of $E A O_{4} \Rightarrow E A O_{2}$ remains the same as above, as well as $E A O_{2} \Rightarrow E A O_{1}$, replacing $C=\sum_{k=i}^{T} C_{k}$ by $C^{\prime}=\sum_{k=i}^{T} C_{k}^{\theta}$.

Proof of Proposition ?? We adopt the same notations as in the proof of Proposition ??.

1. $N F L_{3} \Leftrightarrow N F L_{1}$ : We shall treat here the case where the fixed cost do not depend upon the strategy. The case where the cost depends upon the strategy is an immediate extension, replacing $c_{i}$ with $c_{i}^{\theta^{n}}$ each time it is needed. $E F L_{1} \Rightarrow E F L_{3}$ : There exists a sequence $\left(\theta^{n}\right)_{n \geq 0}$ in $S^{i, B}$ for which $V_{T}^{\theta^{n}} \Leftrightarrow\left(V_{i}^{\theta^{n}}+c_{i}\right) \geq x^{n}+\left(k_{i}^{n} \Leftrightarrow c_{i}\right)$ that converges to $x+\left(k_{i} \Leftrightarrow c_{i}\right) \geq 0, \neq 0$. For $E F L_{3} \Rightarrow E F L_{1}$, we consider the sequence $\tilde{\theta}$ of strategies $\tilde{\theta}^{n}$ null before $i$ and outside $B$ such that for all $n$ in $N$, for all $t \geq i$

$$
\begin{aligned}
\left(\tilde{\theta}^{n}\right)_{t}^{0} & =\left(\theta^{n}\right)_{t}^{0}+\left(\Leftrightarrow c_{i} \Leftrightarrow V_{i}^{\theta^{n}}\right) \text { on } B \text { and } \\
\left(\tilde{\theta}^{n}\right)_{t}^{k} & =\left(\theta^{n}\right)_{t}^{k} \text { for all } k \neq 0
\end{aligned}
$$

It is then easy to check that for all $n$ in $N, \tilde{\theta}^{n}$ is in $S^{i, B}, V_{i}^{\tilde{\theta}^{n}}+c_{i}=0$ and $V_{T}^{\tilde{\theta}^{n}}=V_{T}^{\theta^{n}} \Leftrightarrow\left(V_{i}^{\theta^{n}}+c_{i}\right) \geq x^{n} \rightarrow x \geq 0, \neq 0$ on $B$. Notice that in the case where the cost depends upon the strategy, we use the fact that $c^{\theta}=c^{\theta^{\prime}}$ when $\bar{\theta}=\overline{\theta^{\prime}}$. 
2. $N F L_{2} \Leftrightarrow N F L_{4}: E F L_{2} \Rightarrow E F L_{4}$ is immediate since we can indifferently assume $r \geq 0, \neq 0$ or $r>0$ or there exists a positive real number $\varepsilon$ such that $r \geq \varepsilon$ by considering for all $n$ in $N$ the random variables $\tilde{r}^{n}=r^{n} 1_{r>0}$ and $\hat{r}^{n}=r^{n} 1_{r \geq \varepsilon}$, and the following corresponding strategies $\tilde{\theta}^{n}$ and $\hat{\theta}^{n}$ such that for all $t, \tilde{\theta}_{t}^{n}=\theta_{t}^{n} 1_{r>0}, \hat{\theta}_{t}^{n}=\theta_{t}^{n} 1_{r>\varepsilon}$. For $E F L_{4} \Rightarrow E F L_{2}$, we consider the sequence $\tilde{\theta}$ of strategies $\tilde{\theta}^{n}$ null before $i$ and outside $B$ and such that for all $n$ in $N$, for all $t \geq i$,

$$
\begin{aligned}
\left(\tilde{\theta}^{n}\right)_{t}^{0} & =\left(\theta^{n}\right)_{t}^{0} \Leftrightarrow V_{i}^{\theta^{n}} \Leftrightarrow \varepsilon / 2 \text { on } B \text { and } \\
\left(\tilde{\theta}^{n}\right)_{t}^{k} & =\left(\theta^{n}\right)_{t}^{k} \text { for all } k \neq 0 .
\end{aligned}
$$

Then for all $n$ in $N, \tilde{\theta}^{n}$ is in $W^{i, B}$ and satisfies $\left(V_{T}^{\tilde{\theta}^{n}} \Leftrightarrow V_{i}^{\tilde{\theta}^{n}}\right) 1_{B}=$ $\left(V_{T}^{\theta^{n}} \Leftrightarrow V_{i}^{\theta^{n}}\right) 1_{B}$ so $V_{T}^{\tilde{\theta}^{n}} \geq x^{n} \rightarrow \varepsilon / 2$ on $B$. As $V_{i}^{\tilde{\theta}^{n}}=\Leftrightarrow \varepsilon / 2<0$ on $B$ and $V_{T}^{\tilde{\theta}^{n}}=0$ outside $B$, this completes the proof.

Proof of Corollary ?? immediate using Proposition ??.

\section{Proof of Lemma??}

1. For $N F L_{2} \Rightarrow N F L_{1}$, we prove the implication $N F L_{2} \Rightarrow N F L_{3}$, which is immediate using the fact that $c_{i}>0$ (or that $c_{i}^{\theta^{n}} \geq c_{\mathrm{i}}>0$ in the case where the cost depends upon the strategy) and changing a strategy belonging to $S^{i, B}$ into a strategy belonging to $W^{i, B}$ by proceding like in the proof of Proposition ??.

2. Under Assumption $\mathrm{A}, N F L_{1} \Rightarrow N F L_{2}$ : suppose there is a $F L_{2}$, in the form of a sequence $\left(\theta^{n}\right)_{n \in N}$ of simple strategies like in Definition ??. As we have seen in the proof of Proposition ??, we can indifferently assume that $r \geq 0, \neq 0$ or $r>0$ or there exists a positive real number $\varepsilon$ such that $r \geq \varepsilon$ by considering for all $n$ in $N$ the random variables $\tilde{r}^{n}=r^{n} 1_{r>0}$ and $\hat{r}^{n}=r^{n} 1_{r \geq \varepsilon}$, and the following corresponding strategies $\tilde{\theta}^{n}$ and $\hat{\theta}^{n}$ such that for all $t, \tilde{\theta}_{t}^{n}=\theta_{t}^{n} 1_{r>0}, \hat{\theta}_{t}^{n}=\theta_{t}^{n} 1_{r \geq \varepsilon}$. So there exists a real number $\lambda \geq 1$ such that $\lambda r>C$ where $C$ denotes the real number in the additional Assumption $A$. We consider a sequence $\tilde{\theta}$ of strategies $\tilde{\theta}^{n}$ such that

$$
\begin{gathered}
\tilde{\theta}^{n} \text { is in } S^{i, B} \\
\left(\tilde{\theta}^{n}\right)_{t}^{k}=\lambda\left(\theta^{n}\right)_{t}^{k} \text { for all } k \neq 0 \text { and for all } t \geq i \\
\left(\tilde{\theta}^{n}\right)_{i}^{0}=\lambda\left(\theta^{n}\right)_{i}^{0}+C \Leftrightarrow c_{i} .
\end{gathered}
$$


We then have for all $n$,

$$
V_{i}^{\tilde{\theta}^{n}}=\lambda V_{i}^{\theta^{n}}+C \Leftrightarrow c_{i}
$$

so $V_{i}^{\tilde{\theta}^{n}}+\left(\lambda r^{n} \Leftrightarrow C+c_{i}\right) \leq 0$ with $\left(\lambda r^{n} \Leftrightarrow C+c_{i}\right) \Leftrightarrow c_{i} \rightarrow \lambda r \Leftrightarrow C>0$. We can choose $\tilde{\theta}$ such that for all $n$

$$
V_{T}^{\tilde{\theta}^{n}} \geq \lambda V_{i}^{\theta^{n}} \geq \lambda x^{n} \text { with } \lambda x^{n} \rightarrow \lambda x \geq 0
$$

so the sequence $\tilde{\theta}$ constitutes a free lunch with fixed costs.

3. $N A s F L \Leftrightarrow N F L_{2}: E F L_{2} \Rightarrow E A s F L$ : here again, we can assume that $r$ is (strictly) greater than some positive real number $\varepsilon$ on $B$. The fixed cost at each date is supposed to be bounded $\left(c_{t}<C_{t}\right.$ in the case where the fixed cost does not depend on the strategy and $c_{t}^{\theta}<\mathrm{C}_{\mathrm{t}}$ in the case where the fixed cost depends upon the strategy). Then for all $n$, there exists $\lambda_{n}$ such that $\lambda_{n} \varepsilon$ is greater than the cumulative fixed costs of any simple strategy with the same trading dates as $\theta^{n}$ so that for all $n$, there exists a strategy $\tilde{\theta}_{n}$ in $S^{i, B}$ for which

$$
\begin{aligned}
V_{i}^{\tilde{\theta}^{n}} & =\lambda_{n} V_{i}^{\theta^{n}}+\lambda_{n} \varepsilon \Leftrightarrow \mathrm{C}_{\mathrm{i}} \\
V_{T}^{\tilde{\theta}^{n}} & \geq \lambda_{n} V_{T}^{\theta^{n}} \geq \lambda_{n} x^{n} .
\end{aligned}
$$

We get

$$
\begin{aligned}
\frac{V_{i}^{\tilde{\theta}^{n}}+c_{i}^{\tilde{\theta}^{n}}}{\lambda_{n}} & =V_{i}^{\theta^{n}}+\varepsilon+\frac{c_{i}^{\tilde{\theta}^{n}} \Leftrightarrow \mathrm{C}_{\mathrm{i}}}{\lambda_{n}} \leq \Leftrightarrow r^{n}+\varepsilon \rightarrow \Leftrightarrow r+\varepsilon<0 \\
\frac{V_{T}^{\tilde{\theta}^{n}}}{\lambda_{n}} & \geq x^{n} \rightarrow x
\end{aligned}
$$

$E A s F L \Rightarrow E F L_{2}$ : by investing at each date the fixed cost in the riskless asset, we obtain a sequence $\left(\tilde{\theta}^{n}\right)_{n \geq 0}$ of strategies in $W^{i, B}$. Letting for all $n, \theta^{\prime n}:=\frac{\theta^{n}}{\lambda_{n}}$, we obtain a sequence $\left(\theta^{\prime n}\right)_{n \geq 0}$ of strategies in $W^{i, B}$ such that

$$
\begin{aligned}
& V_{i}^{\theta^{\prime n}} \leq \Leftrightarrow \varepsilon_{i}^{n} \rightarrow \Leftrightarrow \varepsilon_{i}<0 \text { on } B \\
& V_{T}^{\theta^{\prime n}} \geq x^{n} \rightarrow x \geq 0
\end{aligned}
$$


Proof of Theorem ?? First notice that the existence of such a family of probability measures is equivalent to the existence of a family of random variables denoted by $g^{i, B}$ in $L^{\infty}(\Omega, F, P)$ satisfying $g^{i, B} \geq 0, \neq 0$ on $B$ and such that for all $(s, t)$ with $i \leq s \leq t$ and for all $A$ in $F_{s}, E\left[g^{i, B} Z_{t} 1_{A \cap B}\right]=$ $E\left[g^{i, B} Z_{s} 1_{A \cap B}\right]$ : the equivalence is easily obtained by taking $g^{i, B}=d P^{i, B} / d P$ and by defining $P^{i, B}$ by

$$
P^{i, B}(A)=\frac{E\left[g^{i, B} 1_{A \cap B}\right]}{E\left[g^{i, B} 1_{B}\right]} \text { for all } A \text { in } F_{s} .
$$

1) Assume first the existence of such a family of martingale measures and of a sequence $\left(\theta^{n}\right)_{n \in N}$ of strategies such that there exist $i$ in $F$ and $B$ in $F_{i}$ for which for all $n, \theta^{n}$ is in $W^{i, B}$. Let $\left(i=t_{0}^{n}, t_{1}^{n}, \ldots, t_{N_{n}}^{n}=T\right)$ denote the trading dates of the simple strategy $\theta^{n}$. Then using the definition of $V^{\theta^{n}}$, the fact that $\theta^{n}$ is a frictionless self financing strategy, the martingale property of $P^{i, B}$ and the fact that $\theta^{n}$ is null outside $B$, we have for all $n$,

$$
\begin{aligned}
E^{P^{i, B}}\left[V_{T}^{\theta^{n}} \mid F_{i}\right] & =E^{P^{i, B}}\left[\theta_{T}^{n} \cdot Z_{T} \mid F_{i}\right] \\
& =E^{P^{i, B}}\left[\theta_{t_{N_{n}-1}^{n}}^{n} \cdot Z_{T} \mid F_{i}\right] \\
& =E^{P^{i, B}}\left[\theta_{t_{N_{n}-1}^{n}}^{n} \cdot E^{P^{i, B}}\left[Z_{T} \mid F_{t_{N_{n}-1}^{n}}\right] \mid F_{i}\right] \\
& =E^{P^{i, B}}\left[V_{t_{N_{n}-1}^{n}}^{\theta^{n}} \mid F_{i}\right]
\end{aligned}
$$

so that for all $n$,

$$
E^{P^{i, B}}\left[V_{T}^{\theta^{n}} \mid F_{i}\right]=\ldots=E^{P^{i, B}}\left[V_{i}^{\theta^{n}} \mid F_{i}\right]=V_{i}^{\theta^{n}} \text { on } B \text { a.s. } P^{i, B}
$$

and

$$
E^{P^{i, B}}\left[\left(V_{T}^{\theta^{n}} \Leftrightarrow V_{i}^{\theta^{n}}\right) 1_{B} \mid F_{i}\right]=0 .
$$

Then for all $A$ in $F_{i}$, for all $n$ in $N, E\left[g^{i, B}\left(V_{T}^{\theta^{n}} \Leftrightarrow V_{i}^{\theta^{n}}\right) 1_{B \cap A}\right]=0$. Now it is impossible to have $V_{T}^{\theta^{n}} \Leftrightarrow V_{i}^{\theta^{n}} \geq x^{n}$ with $x^{n} \rightarrow_{L^{1}} x \geq \varepsilon$ on $B$ because this would lead to $0=E\left[g^{i, B}\left(V_{T}^{\theta^{n}} \Leftrightarrow V_{i}^{\theta^{n}}\right) 1_{B}\right] \geq E\left[g^{i, B} x^{n} 1_{B}\right]$ and $E\left[g^{i, B} x^{n} 1_{B}\right] \rightarrow E\left[g^{i, B} x 1_{B}\right]>0$-because $g^{i, B}$ is assumed to be bounded-: there exists no frictionless $\varepsilon$-net gain free lunch, which using Proposition ??, completes the proof of the first implication. 
2) Conversely, assume there exists no frictionless strong free lunch. As we have seen in Corollary ??, if $C^{i, B}=\overline{K^{i, B} \Leftrightarrow L_{+}^{1}}$ and

$$
A^{B}=\left\{f \in L_{+}^{1}, \exists \varepsilon>0 \text { such that } f \geq \varepsilon \text { on } B\right\},
$$

the condition of no frictionless strong free lunch is equivalent to the condition that for all $i$ in $F$ and for all $B$ in $F_{i}$,

$$
C^{i, B} \cap A^{B}=\emptyset .
$$

For each fixed $(i, B)$, we apply a strict separation theorem in $L^{1}(\Omega, F, P)$ to the closed convex set $C^{i, B}$ and the compact set $\left\{1_{B}\right\}$ to find $g^{i, B}$ in $L^{\infty}(\Omega, F, P)$ and two real numbers $\alpha$ and $\beta$ with $\alpha<\beta$ such that

$$
\left.g^{i, B}\right|_{C^{i, B}} \leq \alpha<\beta<\left\langle 1_{B}, g^{i, B}\right\rangle .
$$

The random variable $g^{i, B}$ is bounded from above on $C^{i, B}$ and therefore on $L_{-}^{1}$, so $g^{i, B} \geq 0$. As 0 belongs to $C^{i, B}$ and $C^{i, B}$ is a convex cone, we can take $\alpha=0$. Then $\left\langle 1_{B}, g^{i, B}\right\rangle>0$ so $g^{i, B} \neq 0$ on $B$. As 0 belongs to $L_{+}^{1}$, we have $\left.g^{i, B}\right|_{K^{i, B}} \leq 0$ and we even get the equality because $K^{i, B}$ is a vector space. For all $s \geq i$, for all $A$ in $F_{s}$, we consider for all $k$ in $\{1, \ldots, n\}$, the $n$-dimensional random variable $\theta_{s, A, k} \in P^{s, B}$ given by

$$
\begin{aligned}
& \theta_{s, A, k}^{k}=1_{A \cap B} \\
& \theta_{s, A, k}^{l}=0 \text { for all } l \neq k .
\end{aligned}
$$

As $K^{i, B}=\operatorname{Lin}\left\{\theta_{s} \cdot\left(\bar{Z}_{t} \Leftrightarrow \bar{Z}_{s}\right) ; \theta_{s} \in P^{s, B}, i \leq s \leq t\right\}$, we get that for all $k$ in $\{0, \ldots, n\}$, for all $(s, t)$ with $i \leq s \leq t$ and for all $A$ in $F_{s}$, we have

$$
Z_{t}^{k} 1_{A \cap B} \Leftrightarrow Z_{s}^{k} 1_{A \cap B} \in K^{i, B}
$$

Then for all $(s, t)$ with $i \leq s \leq t$, for all $A$ in $F_{s}$ we obtain

$$
E\left[g^{i, B}\left(Z_{t} \Leftrightarrow Z_{s}\right) 1_{A \cap B}\right]=0
$$

or $E\left[g^{i, B} Z_{t} 1_{A \cap B}\right]=E\left[g^{i, B} Z_{s} 1_{A \cap B}\right]$

Proof of Theorem ?? 1) $\Rightarrow$ 2): see Theorem ??. 
2) $\Rightarrow 3$ ): consider $g^{i, B}=d P^{i, B} / d P$.

$3) \Rightarrow 4$ ): let $i$ in $\mp$ and $B$ in $F_{i}$ be fixed. We will write $g$ for $g^{i, B}$ and $\pi$ for $\pi^{i, B}$. We can assume $g=0$ outside $B$. As $g \geq 0, \neq 0$ on $B$, the same is true for the random variable $E\left[g \mid F_{i}\right]$ and there exists a positive real number $\alpha$ such that $P\left(E\left[g \mid F_{i}\right] \geq \alpha\right)>0$. Let $A=\left\{E\left[g \mid F_{i}\right] \geq \alpha\right\}$. Then $A$ belongs to $F_{i}, A \subset B$ and $P(A) \neq 0$. We define an operator $\pi$ on $R^{T, B}$ by

$$
\pi(C)=\frac{E\left[g C \mid F_{i}\right]}{E\left[g \mid F_{i}\right]} 1_{A} \text { for all } C \in R^{T, B} .
$$

The linear operator $\pi$ is linear, continuous and takes values in $R^{i, B}$. If $C \geq 0$, $g C \geq 0$ so $\pi$ is weakly positive. Only the last condition remains to be checked.

Notice first that for all $i \leq s \leq t, E\left[g Z_{t} \mid F_{s}\right] 1_{A}=Z_{s} E\left[g \mid F_{s}\right] 1_{A}$. Now, for all $\theta$ in $W^{i, B}$ with trading dates denoted by $\left(i=t_{0}, t_{1}, \ldots, t_{N}=T\right)$, we have

$$
\begin{aligned}
\pi\left(V_{T}^{\theta}\right) & =\frac{E\left[g \theta_{T} \cdot Z_{T} \mid F_{i}\right]}{E\left[g \mid F_{i}\right]} 1_{A} \\
& =\frac{E\left[\theta_{T_{N-1}} \cdot E\left[g Z_{T} \mid F_{T_{N-1}}\right] \mid F_{i}\right]}{E\left[g \mid F_{i}\right]} 1_{A} \\
& =\frac{E\left[V_{T_{N-1}}^{\theta} E\left[g \mid F_{T_{N-1}}\right] \mid F_{i}\right]}{E\left[g \mid F_{i}\right]} 1_{A}
\end{aligned}
$$

so $\pi\left(V_{T}^{\theta}\right)=\pi\left(V_{T_{N-1}}^{\theta}\right)=\ldots=V_{i}^{\theta} 1_{A}$.

4) $\Rightarrow 1$ ): consider a sequence $\left(\theta^{n}\right)_{n \in N}$ of strategies such that there exist $i$ in $F$ and $B$ in $F_{i}$ such that for all $n, \theta^{n}$ is in $W^{i, B}$. For all $n$ in $N$, we then have $1_{A} \pi^{i, B}\left(V_{T}^{\theta^{n}} \Leftrightarrow V_{i}^{\theta^{n}}\right)=0$. Now it is impossible to have $V_{T}^{\theta^{n}} \Leftrightarrow V_{i}^{\theta^{n}} \geq x^{n}$ with $x^{n} \rightarrow_{L^{1}} x \geq \varepsilon$ on $B$ because this would lead to $0=1_{A} \pi^{i, B}\left(V_{T}^{\theta^{n}} \Leftrightarrow V_{i}^{\theta^{n}}\right) \geq$ $1_{A} \pi^{i, B}\left(x^{n}\right)$ because $\pi^{i, B}$ is linear and weakly positive; as $\pi^{i, B}$ is continuous, $1_{A} \pi^{i, B}\left(x^{n}\right) \rightarrow 1_{A} \pi^{i, B}(x) \geq 1_{A} \pi^{i, B}\left(\varepsilon 1_{A}\right) \geq \varepsilon 1_{A}$ because $1_{A} \pi^{i, B}\left(1_{A}\right)=1_{A}$ : a contradiction.

1) $\Leftrightarrow 5)$ : see Lemma ??-

Proof of Theorem ?? 1) $\Rightarrow$ 2) is immediate.

$2) \Rightarrow 3$ ): using a strict separation theorem exactly like in the proof of our main theorem, we get that there exists a random variable $Z$ in $L^{\infty}(\Omega, F, P)$ 
such that

$$
\zeta \in K, \alpha \in L_{+}^{1} \sup _{\zeta \in K, \alpha \in L_{+}^{1}} E[Z(\zeta \Leftrightarrow \alpha)]<c E\left[Z 1_{\Omega}\right]
$$

As 0 belongs to $K$, replacing $\alpha$ by $a \alpha$ with $a \geq 0$, we get $Z \geq 0$. We have $Z \neq 0$, because if $Z=0$, then $c E\left[Z 1_{\Omega}\right]=0$ and we would get $0<0$. Taking $\alpha=0$, we obtain $\sup _{\zeta \in K} E[Z \zeta]<c E\left[Z 1_{\Omega}\right]<\infty$.

$3) \Rightarrow 1$ ): Suppose 1) does not hold; then there exists $\eta$ in $L^{1}, \eta>0$ such that for all $n$ in $N^{*}, n \eta$ is in $\overline{K \Leftrightarrow L_{+}^{1}}$. As $n \eta$ is in $\overline{K \Leftrightarrow L_{+}^{1}}$, for all $n$ in $N^{*}$, there is a sequence $\left(\eta_{n}^{p}\right)_{p \in N}$ such that $n \eta=\lim _{p} \eta_{n}^{p}$ and for all $n$, for all $p$, there is $\zeta_{n}^{p}$ in $K$ satisfying $\eta_{n}^{p} \leq \zeta_{n}^{p}$. Then $E\left[Z \eta_{n}^{p}\right] \leq E\left[Z \zeta_{n}^{p}\right]$ and as $Z$ is uniformly bounded, $E\left[Z \eta_{n}^{p}\right] \rightarrow_{p} E[Z n \eta]=n E[Z \eta] \rightarrow \infty$ so condition 3$)$ is not satisfied.

Proof of Corollary ?? 1) $\Rightarrow 2$ ) is obvious. 2) $\Rightarrow 3$ ) and 3) $\Rightarrow$ 1) are in the proof of our main theorem.

Proof of Proposition ??: we have assumed that there is no arbitrage in the primitive market, so that if two frictionless self financing strategies $\theta$ and $\theta^{\prime}$ are such that $V_{T}^{\theta}=V_{T}^{\theta^{\prime}}$, then $V_{0}^{\theta}=V_{0}^{\theta^{\prime}}$. We define on $M$ a linear functional $l$ given by $l\left(V_{T}^{\theta}\right)=V_{0}^{\theta}$. Now it is easy to see that for all $B$ in $M$,

$$
\lim _{\lambda \rightarrow+\infty} \frac{\pi_{s}(\lambda B)}{\lambda}=\lim _{\lambda \rightarrow+\infty} \frac{\Leftrightarrow \pi_{s}(\Leftrightarrow \lambda B)}{\lambda}=l(B)
$$

Since there is no arbitrage, we must have $p(B) \geq \Leftrightarrow p(\Leftrightarrow B)$ so that

$$
\Leftrightarrow \pi_{s}(\Leftrightarrow B) \leq \Leftrightarrow p(\Leftrightarrow B) \leq p(B) \leq \pi_{s}(B),
$$

and the price functional $p$ can be written as the sum of a continuous linear functional and a fixed cost, i.e., for all $B, p(B)=l(B)+c(B)$ where $\frac{c(\lambda B)}{\lambda} \rightarrow_{\lambda \rightarrow \infty} 0$.

If we assume that $p(\lambda x)<\lambda[p(x)]$, then the fixed cost is nonnegative; moreover, if we assume that there exists $\varepsilon>0$, such that for a large enough $\lambda, p(\lambda x)<\lambda[p(x) \Leftrightarrow \varepsilon]$, then the fixed $\operatorname{cost} c$ is greater than or equal to this positive constant $\varepsilon$. Notice that $c(B):=p(B) \Leftrightarrow l(B) \leq \pi_{s}(B) \Leftrightarrow l(B) \leq C$.

Consequently, the fair price $p(B)$ associated with any attainable contingent claim $B$ is given by

$$
p(B)=E^{P^{*}}[B]+\text { a fixed cost }
$$


where $P^{*}$ is any absolutely continuous martingale measure.

Proof of Proposition ?? Since $\pi$ is a sublinear lower semicontinuous functional defined on a vector space, it can be written as the supremum of all continuous linear functionals lying below it,

$$
\pi(x)=\sup _{\tilde{l} \leq \pi, \tilde{l} \text { cont. lin. funct. }} \tilde{l}(x) .
$$

We first show that $\tilde{l}\left(V_{T}^{\theta}\right)=V_{0}^{\theta}$ for $\theta \in W^{0, \Omega}$. Since $\tilde{l}+c$ lies below the superreplication price, it satisfies $\tilde{l}\left(V_{T}^{\theta}\right)+c\left(V_{T}^{\theta}\right) \leq V_{0}^{\theta}+C$, so that for all positive real number $\lambda, \frac{\tilde{l}\left(V_{T}^{\lambda \theta}\right)}{\lambda}+\frac{c\left(V_{T}^{\lambda \theta}\right)}{\lambda} \leq \frac{V_{0}^{\lambda \theta}}{\lambda}+\frac{C}{\lambda}$ and letting $\lambda$ go to $\infty$, $\tilde{l}\left(V_{T}^{\theta}\right) \leq V_{0}^{\theta}$. In the same way, we obtain $\tilde{l}\left(\Leftrightarrow V_{T}^{\theta}\right) \leq \Leftrightarrow V_{0}^{\theta}$, which gives us $\tilde{l}\left(V_{T}^{\theta}\right)=V_{0}^{\theta}$.

It is easy to see that $\tilde{l}$ is weakly positive. Indeed, for all $B \leq 0, \pi_{s}(B) \leq 0$ so that $\tilde{l}(B) \leq 0$ and for all $B \geq 0, \tilde{l}(B) \geq 0$.

According to the following Lemma, there is a one-to-one correspondence between absolutely continuous martingale measures with bounded density $P^{*}$ and weakly positive continuous linear functionals $\tilde{l}$ such that $\tilde{l}\left(V_{T}^{\theta}\right)=V_{0}^{\theta}$. The functional $\pi$ can therefore be written in the form $\pi(x)=\sup _{P^{*} \in K} E^{P^{*}}[x]$ where $K$ denotes a convex subset of the set of all absolutely continuous martingale measures.

Proof of the Lemma Let $P^{*}$ be such that for all $B$ in $F, P^{*}(B)=$ $p\left(1_{B}\right)$. As there exists a strategy in $W^{0, \Omega}$ with terminal value $V_{T}^{\theta}=1_{\Omega}$ and initial value $V_{0}^{\theta}=1$, we have $p\left(1_{\Omega}\right)=1$; as $p$ is assumed to be weakly positive and sublinear, $P^{*}$ takes values in $[0,1]$; as -according to the remark preceding the lemma- $p$ is continuous and linear, $P^{*}$ is a probability measure. As $p(0)=0$, we get $P^{*}<<P$. As $p$ is a continuous linear functional on $L^{1}(\Omega, F, P)$, there exists $g$ in $L^{\infty}(\Omega, F, P)$ such that for all $b$ in $L^{1}(\Omega, F, P)$, $p(b)=E[g b]$. Then $E^{P^{*}}[b]=E[g b]=p(b)$ so for all strategy $\theta$ in $W^{0, \Omega}$, $E^{P^{*}}\left[V_{T}^{\theta}\right]=p\left(V_{T}^{\theta}\right)=V_{0}^{\theta}$ so for all $(s, t)$ with $s \leq t$,

$$
E^{P^{*}}\left[\left(Z_{t} \Leftrightarrow Z_{s}\right) 1_{A}\right]=0 \text { for all } A \text { in } F_{s}
$$

or $Z$ is a $P^{*} \Leftrightarrow$ martingale.

Conversely let $p$ be defined by $p(b)=E^{P^{*}}[b]$ for all $b$ in $L^{1}(\Omega, F, P)$. Then $p$ is linear, continuous because $d P^{*} / d P$ belongs to $L^{\infty}(\Omega, F, P)$, weakly 
positive because, as $P^{*}$ is absolutely continuous with respect to $P$, we have $g=d P^{*} / d P \geq 0$. Finally, for all strategy $\theta$ in $W^{0, \Omega}, p\left(V_{T}^{\theta}\right)=E^{P^{*}}\left[V_{T}^{\theta}\right]=$ $V_{0}^{\theta}$.

Proof of Theorem ?? $\quad(3) \Leftrightarrow(4)$ is equivalent to $(1) \Leftrightarrow(4)$ for $i=0$ and $B=\Omega$ in Theorem ??.

$(2) \Leftrightarrow(4):(4) \Rightarrow(2)$ : we take $\tilde{r}^{n}=r^{n}+\varepsilon$ that converges to $r+\varepsilon>0$ and $\tilde{x}^{n}=x^{n}$. (2) $\Rightarrow(4)$ : there is a real number $\lambda \geq 1$ such that $\lambda r>C$. We get $V_{0}^{\lambda \theta^{n}}+c\left(V_{T}^{\lambda \theta^{n}}\right) \leq V_{0}^{\lambda \theta^{n}}+C \leq C \Leftrightarrow \lambda \tilde{r}^{n}$ with $C \Leftrightarrow \lambda \tilde{r}^{n} \rightarrow C \Leftrightarrow \lambda r<0$ and $V_{T}^{\lambda \theta^{n}} \geq \lambda \tilde{x}^{n}$ with $\lambda \tilde{x}^{n} \rightarrow \lambda x \geq 0$ so that $\left(V_{T}^{\lambda \theta^{n}}\right)_{n \in N}$ is a free lunch for $(M, p)$. $(1) \Leftrightarrow(3):(1) \Rightarrow(4)$ : suppose there is a free lunch $\left(\theta^{n}\right)_{n \in N}$ : we can assume $V_{T}^{\theta^{n}} \geq \tilde{x}^{n}+\varepsilon$ for some $\varepsilon>0, V_{0}^{\theta^{n}} \leq \Leftrightarrow C$. Since $\left(r^{*}, m^{*}\right)$ is optimal, and $r^{*}+c\left(V_{T}^{\theta^{n}+\theta^{*}}\right)+V_{0}^{\theta^{n}+\theta^{*}} \leq r^{*}+V_{0}^{\theta^{*}}+V_{0}^{\theta^{n}}+C \leq \Leftrightarrow c\left(V_{T}^{\theta^{*}}\right) \leq 0$, we have $\left(r^{*}, m^{*}\right) \succeq\left(r^{*}, V_{T}^{\theta^{n}+\theta^{*}}\right)$. So for all $n,\left(r^{*}, m^{*}\right) \succeq\left(r^{*}, \tilde{x}^{n}+\varepsilon+V_{T}^{\theta^{*}}\right)$ because $\succeq$ is increasing and $V_{T}^{\theta^{n}} \geq \tilde{x}^{n}+\varepsilon$ which gives, using the fact that preferences are continuous, $\left(r^{*}, m^{*}\right) \succeq\left(r^{*}, x+\varepsilon+m^{*}\right)$ : a contradiction.

$(3) \Rightarrow(1)$ : We define $\succeq$ by $(r, x) \succeq\left(r^{\prime}, x^{\prime}\right) \Leftrightarrow r+\pi(x) \geq r^{\prime}+\pi\left(x^{\prime}\right)$. Then one can show that $\succeq$ belongs to $A$ and that $(0,0)$ is optimal.

Proof of Theorem ?? We proceed exactly like in the proof of Theorem ?? 


\section{References}

[1] Ansel, J.P, Stricker, C (1990): Quelques remarques sur un théorème de Yan. Sém. de probabilités. Lecture notes in Mathematics XXIV Springer-Verlag, New-York, 226-74.

[2] Back, K., Pliska, S.R. (1990): On the fundamental theorem of asset pricing with an infinite state space. J. Math. Econ. 20, 1-33.

[3] Bensaid, B., Lesne, J.P., Pages, H., Scheinkman, J. (1992): Derivative asset pricing with transaction costs. Math. Fin. 2, 63-86.

[4] Black, F., Scholes, M. (1973): The pricing of options and corporate liabilities. J. Pol. Econ. 81, 637-54.

[5] Clark, S. (1993): The valuation problem in arbitrage price theory. J. Math. Econ. 22, 463-78.

[6] Constantinides, G. (1986): Capital market equilibrium with transaction costs. J. Pol. Econ. 94, 842-62.

[7] Cox, J., Ross, S. (1976): The valuation of options for alternative stochastic processes. J. Fin. Econ. 3, 145-66.

[8] Cvitanic, J., Karatzas, I. (1993): Hedging contingent claims with constrained portfolios. Ann. App. Probab. 3, 652-81.

[9] Cvitanic, J., Karatzas, I. (1996): Hedging and portfolio optimization under transaction costs: a martingale approach. Math. Fin. 6, 133-166.

[10] Dalang, R.C., Morton, A., Willinger, W. (1989): Equivalent martingale measures and no arbitrage in stochastic securities market models. Stochastics and Stochastic Rep. 29, 185-202.

[11] Debreu, G. (1959): Theory of value, Wiley, New York.

[12] Delbaen, F., Schachermayer, W. (1994): A general version of the fundamental theorem of asset pricing. Math. Ann. 300, 463-520.

[13] Delbaen, F., Schachermayer, W. (1995): The existence of absolutely continuous local martingale measures. Ann. App. Probab. 5, 926-45. 
[14] Delbaen, F., Schachermayer, W. (1998): The fundamental theorem of asset pricing for unbounded stochastic processes. Math. Ann. 312, 215 -250 .

[15] Duffie, D., Huang, C. (1986): Multiperiod security markets with differential information: martingales and resolution times. J. Math. Econ. 15, 283-303.

[16] Duffie, D., Sun, T. (1990): Transaction costs and portfolio choice in a discrete-continuous time setting. J. Econ. Dyn. and Control, 14, 35-51.

[17] Dybvig, P., Ross, S. (1986): Tax clienteles and asset pricing. J. Fin. 41, 751-62.

[18] Dybvig, P., Ross, S. (1987): Arbitrage, in: Eatwell, J., Milgate, M., and Newman,P., eds., The new Palgrave: A dictionary of Economics, vol.1 (Macmillan, London) 100-106.

[19] Grossman, S., Laroque, G. (1990): Asset pricing and optimal portfolio choice in the presence of illiquid durable consumption goods. Econometrica $\mathbf{5 8}, 25-51$.

[20] Harrison, M., Kreps, D. (1979): Martingales and arbitrage in multiperiod security markets. J. Econ. Theory 20, 381-408.

[21] Harrison, M., Pliska, S. (1981): Martingales and stochastic integrals in the theory of continuous trading. Stochastic Processes Appl. 11, 215260 .

[22] He, H., Pearson, N. (1991): Consumption and portfolio policies with incomplete markets and short-sale constraints: the infinite dimensional case. J. Econ. Theory 5, 259-304.

[23] Hindy, A. (1995): Viable prices in financial markets with solvency constraints. J. Math. Econ. 24, 105-36.

[24] Jouini, E., Kallal, H. (1995a): Martingales and arbitrage in securities markets with transaction costs. J. Econ. Theory 66, 178-97.

[25] Jouini, E., Kallal, H. (1995b): Arbitrage in securities markets with short sales constraints. Math. Fin. 5, 197-232. 
[26] Jouini, E., Kallal, H. (1999): Viability and equilibrium in securities markets with frictions. Math. Fin. 9, 275-92.

[27] Kreps, D. (1981): Arbitrage and equilibrium in economies with infinitely many commodities. J. Math. Econ. 8, 15-35.

[28] Magill, M., Constantinides, G. (1976): Portfolio selection with transaction costs. J. Econ. Theory 13, 245-63.

[29] Morton, A., Pliska, S. (1995): Optimal portfolio management with fixed transaction costs. Math. Fin. 5, 337-56.

[30] Pham, H., Touzi, N. (1996): The fundamental theorem of asset pricing with cone constraints. Working paper. Crest.

[31] Prisman, E. (1986): Valuation of risky assets in arbitrage-free economies with frictions. J. Fin. 41, 545-60.

[32] Ross, S. (1987): Arbitrage and martingales with taxation. J. Pol. Econ. 95, 371-93.

[33] Stricker, C. (1990): Arbitrage et lois de martingale. Ann. Inst. Henri Poincaré 26, 451-460.

[34] Taksar, M., Klass, M., Assaf, D. (1988): A diffusion model for optimal portfolio selection in the presence of brokerage fees. Math. Oper. Res. 13, 277-94.

[35] Yan, J.A. (1980): Caractérisation d'une classe d'ensembles convexes de $L^{1}$ ou $H^{1}$. Sém. de Probabilités. Lecture notes in Mathematics XIV 784, 220-22. 